\title{
Design and Performance Evaluation of WDM/TDMA-Based MAC Protocol in AWG-Based WDM-PON
}

\author{
Kyeong-Eun Han ${ }^{1}$, Yang He ${ }^{1}$, Seung-Hyun Lee ${ }^{1}$ \\ Biswanath Mukherjee ${ }^{2}$, and Young-Chon Kim ${ }^{1}$ \\ 1 Chonbuk National University, Jeonju 561-756, Korea \\ $\{$ kehan, yckim\}@chonbuk.ac.kr \\ 2 University of California. Davis, CA 95616, USA
}

\begin{abstract}
In this paper, we propose a MAC protocol for a passive optical network (PON) employing wavelength-division multiplexing (WDM) with arrayed-waveguide gratings (AWGs) performing routing functionality at the remote node. In proposed MAC protocol, we employ a Request/Permit based MAC protocol using multipoint control protocol (MPCP). It is simulated using OPNET and their performances are evaluated in terms of bandwidth utilization and queuing delay.
\end{abstract}

\section{Introduction}

A PON is a passive broadcast optical network consisting of an optical line terminal (OLT) at the central office connected to many optical network units (ONUs), through a passive optical device (for distribution/collection) located at a remote node $(\mathrm{RN})$. Although a PON provides the necessary improvements, simplicity, scalability and low cost of implementation, they do not provide a scalable solution for access networks as the number of users is limited by the splitter attenuation. The AWG-based WDM-PON is the new technology to solve these problems. Network costs and complexity can be significantly reduced by using arrayed-waveguide gratings (AWGs) which support the wavelength-selective routing function [1]. Thus, these networks are intrinsically transparent to the channel capacity, do not suffer power splitting losses and enhance the reliability and privacy. In order to provide efficient resource allocation and use ethernet frame for carrying IP traffic more easily in WDM-PON, a appropriate MAC protocol is required. Therefore, in this paper, we propose a MAC protocol employing a Request/Permit based on MPCP for dynamic bandwidth allocation (DBA).

\section{AWG based WDM-PON Architecture}

The used WDM-PON architecture is shown in Figure 1. Downstream traffic is transmitted from OLT through two stages of AWGs: Stage-1 and Stage-2. For 
upstream traffic we employ a separate fiber wherein the WDM signals (packets) from Stage-2 AWGs are multiplexed using TDMA. At each cluster of upstream, a given ONU (say $n t h$ ONU) transmits the data on a wavelength which is being used to receive downstream data in its immediately preceding ONU (say $(n-1) t h$ ONU). For downstream transmission, 32 wavelengths from any input port are demultiplexed into 8 output ports by using free spectral range (FSR) property. Thus each output port in Stage-1 has 4 wavelengths from an input port. In all there are 16 wavelengths from 4 input ports which are all even or odd indices of wavelengths. As mentioned earlier, combiner at Stage-2 combines 32 ONUs with different wavelengths and 32 wavelengths (combined at Stage-2) are recombined by the combiner at Stage-1. This results in the collision in this part. Therefore, to avoid the collision and provide an efficient bandwidth allocation among ONUs sharing with same wavelength, an appropriate MAC protocol is required.

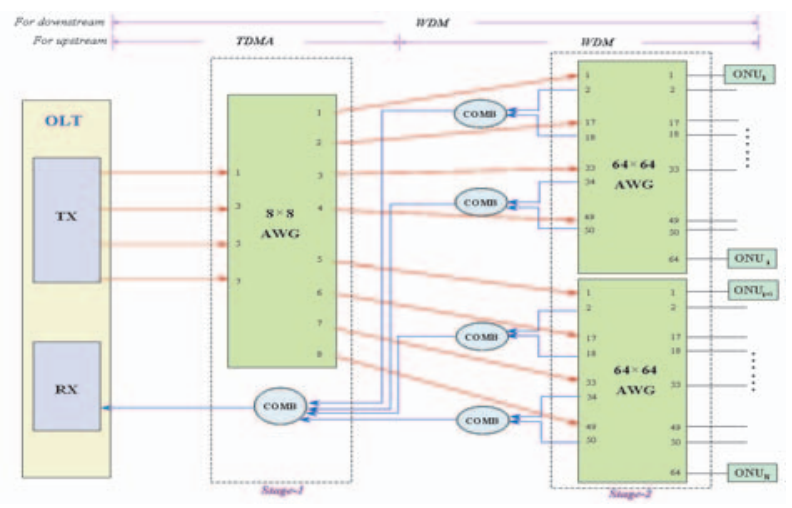

Fig. 1. WDM PON system architecture

\section{Proposed MAC Protocol}

In this section, we describe our MAC protocol, which controls the access of shared wavelengths for upstream transmission as well as downstream one for controlling upstream. In particular a Request/Permit based MAC protocol employing MPCP is used. We also use ethernet container for control and data packets. Because using one, the network can easily carry IP packets and can use ubiquitous/cheap hardware with the desired scalability. In MPCP the procedure for DBA relies on two control messages: GATE and REPORT. GATE is used by OLT on downstream to grant the bandwidth to ONUs for both discovery and normal transmission. REPORT is used by ONUs to inform the ONU's queue information to the OLT. GATE and REPORT are transmitted for every frame periodically. Fig. 2 (a) and (b) shows the down/upstream frame format respectively.

Fig. 3 shows the exchange procedure of control message for registration and DBA respectively. In fig. 3-(a) first, the OLT broadcasts GATE containing the 


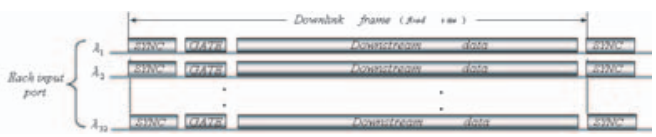

(a)

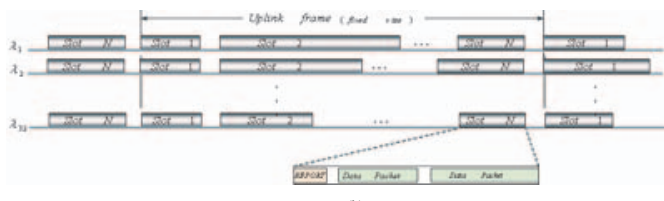

(b)

Fig. 2. (a)Downstream and (b)Upstream frame format

information of wavelength, input port number and allocated bandwidth for registration. Upon receiving GATE, active ONUs reply to OLT with the REGISTERREQEUST. If this message is successfully received, the OLT searches the ONU-id from table and allocates it through the REGISTER. Finally, the OLT and ONU confirm to complete this procedure as exchanging GATE and REGISTER-ACK. In fig. 3(b) the OLT broadcasts GATE to all ONUs based on the mapping table. Each ONU (received the GATE from OLT) send the REPORT along with their own data to be allocated from OLT over a specific channel. After receiving the REPORT from all ONUs, OLT schedules based on requested bandwidth for each ONU sharing with the same channel and transmits the allocation results through GATE.
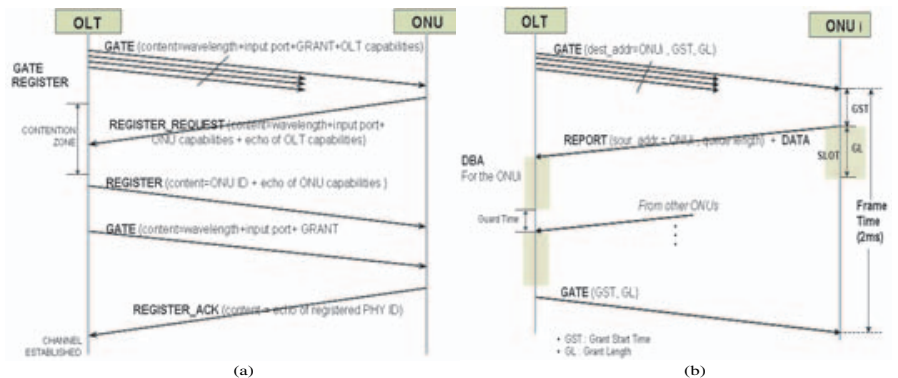

Fig. 3. Procedure of (a) registration and (b) dynamic bandwidth allocation

\section{Performance Evaluation}

We simulate and evaluate the performance of proposed MAC protocol using OPNET under the hot spot ratio (h). We assume 128 ONUs, 32 wavelengths, 96 bits inter-packet gap (IPG) and $1 \mu s$ guard time for simulation. We also assume the fixed frame size $(2 \mathrm{~ms})$ and $1 \mathrm{Gbps}$-channel capacity. Fig. 4 present the plots of utilization and queuing delay vs. offered load for the static and dynamic bandwidth allocation. 


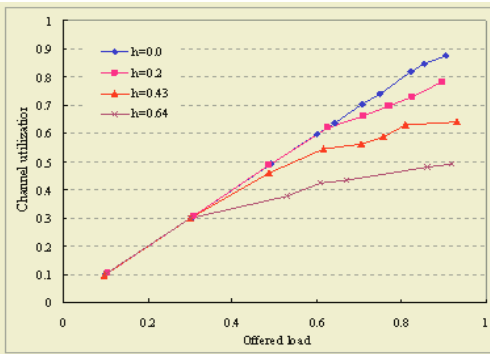

(a) Static TDMA

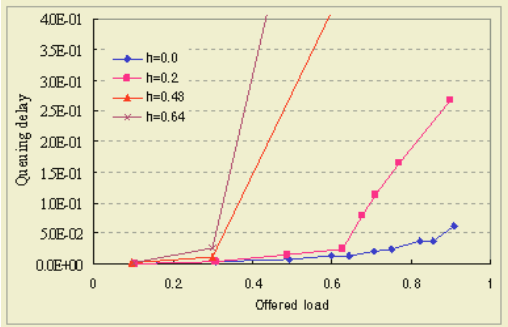

(c) Static TDMA

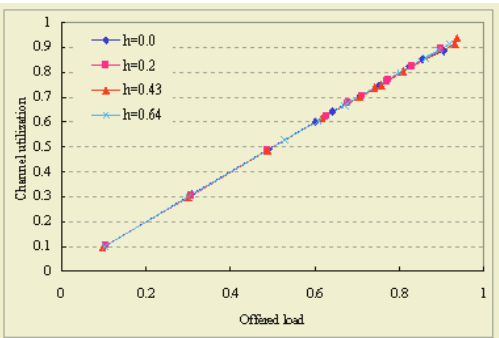

(b) Dynamic TDMA

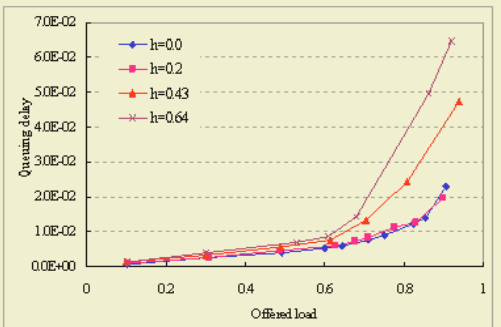

(b) Dynamic TDMA

Fig. 4. Channel utilization and queuing delay both MAC protocols

\section{Conclusion}

We proposed the MAC protocol based on a Request/Permit for DBA. It employs a minimum bandwidth guaranteed algorithm. The simulation results indicated the proposed MAC protocol can significantly improve the bandwidth utilization and queuing delay regardless of hot spot ratio.

\section{Acknowledgement}

This work is supported by IITA and Korea Industrial Technology Foundation(KOTEF), Republic of Korea.

\section{References}

1. Guido Mario, "Design and Cost Performance of the Multistage WDM-PON Access Networks," J. Lightwave Technology, vol.18, pp.125-143, February 2000. 